\title{
Blood Pressure Control and Correlation Dimension
}

\author{
S Eyal, S Akselrod \\ Tel-Aviv University, Tel-Aviv, Israel
}

\begin{abstract}
A simple continuous nonlinear model of the human cardiovascular system (CVS) is investigated while focusing on the simulated achieved blood pressure (BP) signal. The beat-to-beat version of this model, introduced by DeBoer et. al. was a simplified linearized version. We present a modified model, which allows investigating the nonlinear dynamics of the CVS and the effect of variations in several physiological parameters on the correlation dimension, $D_{2}$. The simulation reveals nonlinear features such as bifurcation, due to variations in the $\alpha$-sympathetic gain and the respiratory term. In addition, we studied the limitations of calculating $D_{2}$ in the presence of noise. The $D_{2}$ values obtained from the simulated BP signals are similar to values obtained in our previous experimental animal studies. Our results emphasize that nonlinearity in cardiovascular control is indeed a fundamental feature of the system.
\end{abstract}

\section{Introduction}

Recently there has been a growing interest in using nonlinear analysis tools for investigating the characteristics of the cardiovascular control system. In this work nonlinear tools were used to study pseudo-continuous blood pressure (BP) signals that were produced by a simple nonlinear model of the short-term control over the cardiovascular system (CVS). The model that was used was a modified version of the beat-to-beat model that has been previously proposed by DeBoer et. al. [1]. Though the original model was based on nonlinear equations, it was studied after linearization.

An important tool for characterizing nonlinear properties of systems is the correlation dimension $\left(D_{2}\right)$. Yet, in contrast with linear analysis tools and despite many recent studies, the physiological meaning of $D_{2}$ is to a large extent unclear.

The goal of this work was to study the physiological meaning of $D_{2}$ and to investigate the effect of various physiological parameters as well as noise sources, on the dynamical behavior of the CVS as they are revealed by the behavior of $D_{2}$.

\section{Method}

Model Design: The beat-to-beat model consists of a set of difference equations [1,3]. These equations describe the temporal evolution of the following state variables: systolic pressure $(S)$, diastolic pressure $(D), \mathrm{RR}$ intervals $(I)$, and arterial decay time constant $(T)$.

The diastolic pressure $\left(D_{i}\right)$ of each beat is expressed in terms of the previous beat parameters (denoted $X_{i-1}$ ), in accordance with the Windkessel properties of the systemic arterial tree [1]:

$$
D_{i}=S_{i-1} \exp \left(-(2 / 3) I_{i-1} / T_{i-1}\right)
$$

The variations of the systolic pressure are governed by the contractile properties of the myocardium and the mechanical effect of respiration, according to:

$$
S_{i}=D_{i}+\gamma I_{i-1}+C_{1}+A \sin \left(2 \pi f\left(t_{i} / 1000\right)\right)+N_{P P i}
$$

where $\gamma$ and $C_{1}$ are constants, $A$ and $f$ respectively are the amplitude and frequency of an oscillatory term reflecting the effect of respiration, $t_{i}$ is the time elapsed from the beginning of the simulation to the current systole (measured in msec) and $N_{P P i}$ is a Gaussian noise source affecting the pulse pressure.

The sigmoidal nature of the baroreceptors sensitivity is accounted for by defining an effective systolic pressure $\left(S^{\prime}\right)(3)$ [1], upon which the control over the heart rate (4) and peripheral resistance (6) depends.

$$
S_{i}^{\prime}=S_{0}+18 \arctan \left(\left(S_{i}-S_{0}\right) / 18\right)
$$

where $S_{0}$ is a constant.

The control over the heart rate is a combination of two mechanisms: the vagal control and the comparatively slower $\beta$-sympathetic control, both defined by their respective gain $\left(G_{v}=18 \mathrm{msec} / \mathrm{mmHg}, G_{\beta}=18 \mathrm{msec} / \mathrm{mmHg}\right)$ and delay $\left(\tau_{v}=0\right.$ beat, $\tau_{\beta}=4$ beat $)$ :

$$
I_{i}=G_{v} S_{i-\tau_{v}}^{\prime}+G_{\beta} F\left(S^{\prime}, \tau_{\beta}\right)+C_{2}+N_{R R I i}
$$

where $\mathrm{S}_{\mathrm{i}}^{\prime}$ is the above defined effective systolic pressure, $C_{2}$ is a constant, $N_{R R I i}$ is the Gaussian noise affecting the $\mathrm{RR}$ intervals, and $F\left(S^{\prime}, \tau\right)$ is a linear weighted sum of five effective systoles centered at $i-\tau$ 


$$
F\left(S^{\prime}, \tau\right)=\left(S_{i-\tau-2}^{\prime}+2 S_{i-\tau-1}^{\prime}+3 S_{i-\tau}^{\prime}+2 S_{i-\tau+1}^{\prime}+S_{i-\tau+2}^{\prime}\right) / 9
$$

The arterial decay time is controlled by the $\alpha$ sympathetic control loop, which is given in terms of its gain $\left(G_{\alpha}=18 \mathrm{msec} / \mathrm{mmHg}\right)$ and delay $\left(\tau_{\alpha}=4\right.$ beat $)$ :

$$
T_{i}=C_{3}-G_{\alpha} F\left(S^{\prime}, \tau_{\alpha}\right)+N_{\tau i}
$$

where $C_{3}$ is a constant and $N_{\tau i}$ is the Gaussian noise added to the arterial decay time.

The constants of the model $\left(C_{1,}, C_{2}, C_{3}, \gamma\right.$ and $\left.S_{0}\right)$ are determined according to the operating point chosen for each state variable. The values we have used for the operating point are considered to be normal human values: $120 \mathrm{mmHg}$ and $75 \mathrm{mmHg}$ for systolic and diastolic pressure respectively, $800 \mathrm{msec}$ for average RR intervals and $1134 \mathrm{msec}$ for the average arterial time constants.

It is important to note that we have performed our simulations with the nonlinear equations in their original format without any linearization.

Simulation of continuous BP signal: We have used an interpolation procedure in order to produce a continuous BP sequences from the beat-to-beat state variables. Each beat in these signals comprised a linear rise in pressure and an exponential decay of it, in which $T_{i}$ serves as the exponential decay constant. The duration of each beat was set by its $I_{i}$. The ratio between the duration of the linear uprise and the duration of the exponential decay was constant throughout each simulation. This 'continuous' time signal was then digitized at a frequency of $25 \mathrm{~Hz}$, which produces 20 points for each heart beat under normal physiological conditions. Each final BP signal consists of 10,000 points in a steady state condition.

Calculation of the $D_{2}$ : Then for each synthesized 'continuous' BP signal we have calculated the correlation dimension, $D_{2}$, as suggested by Grassberger and Procaccia [5]. The calculation requires a careful choice of several parameters. In order to be able to compare between $D_{2}$ values that were obtained from simulated $\mathrm{BP}$ traces and from experimental traces, exactly the same calculating protocol was used as in [4]. A detailed discussion regarding the specific choice of parameters can be found there [4].

We will elaborate here only on the embedding dimensions, $n$, that were used. $D_{2}(n)$ should reach a plateau as a function of the embedding dimension. We have used an acceptance criterion for the $D_{2}$ based on this plateau. A weighted average over the $D_{2}(n)$, with $1 /$ variance being the weight and the corresponding standard deviation (SD) were calculated over the $D_{2}(n)$ obtained for embedding dimensions $n=6$ to 10 [4], producing $D S_{2} \pm \Delta D S_{2}$. The convergence of $D_{2}(n)$ as well as the validity of the $D S_{2}$ was determined by requiring that $\Delta D S_{2} / D S_{2}<1.5 \%$ [4]. The threshold of $1.5 \%$ was chosen after examining many $D_{2}(n)$ graphs.
For each set of parameters of the simulation, we have produced five similar signals in which the only change was the noise signal that we added to the simulation. In these cases an average over the five $D S_{2}$ was performed, to produce the actual $D_{2} \pm \Delta D_{2}$ for the set of parameters. Only valid $D S_{2}$, that passed the convergence test, were added to the average.

Our software for calculating $D_{2}$ was tested successfully on signals with well-known $D_{2}$ values (Henon map, Lorenz model, and Mackey-Glass).

\section{Results}

The default parameters without noise and without the respiration term at (2) produced a value of $D_{2}=1.0091 \pm 0.0004$ for the simulated BP traces.

Effect of Noise: The three noise sources (equations 2, 4 and 6) were added to the simulation in a variety of amplitude combinations. As a result of the addition of these noises, repeated simulations with the same set of parameters resulted in variation in the correlation dimension of each signal, $D S_{2}$. These variations were higher than the error within each calculation, $\Delta D S_{2}$. Therefore each set of parameters was simulated and studied 5 times, and $D S_{2}$ was averaged to produce $D_{2}$.

An example of the influence of the level of noise on the relative error of $D S_{2}$ is shown in the simulations in Figure 1, in which we added only $\mathrm{N}_{\mathrm{pp}}$ to equation 2 . $D S_{2}$ showed similar behavior when the other noise

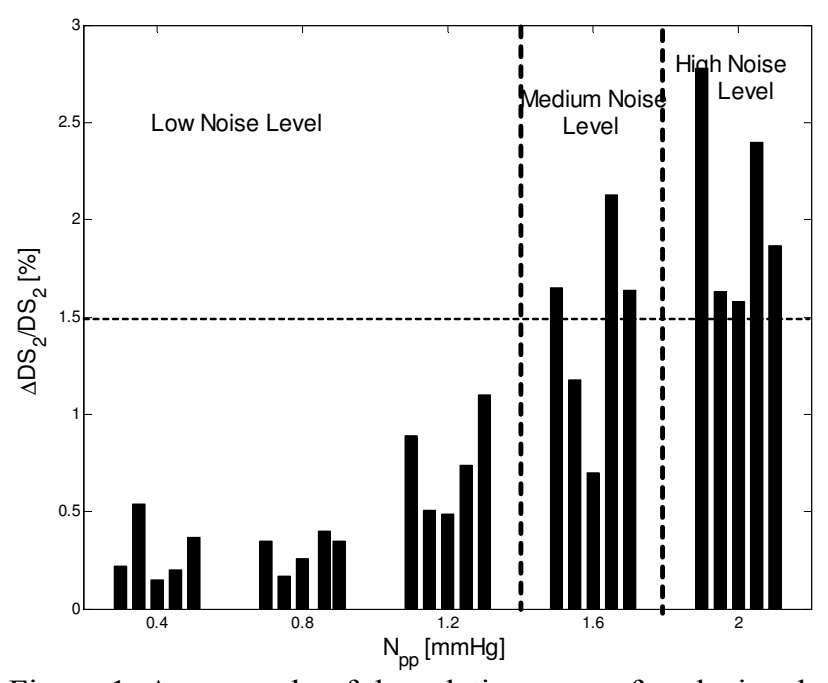

Figure 1. An example of the relative error of each signal, $\Delta D S_{2} / D S_{2}$, as a function of the level of noise added to the pulse pressure, $\mathrm{N}_{\mathrm{pp}}$ in equation 2 . 
sources, $N_{R R I}$ and $N_{\tau}$, were added, either separately, or as a combination of two or three noise sources.

Noise with small amplitude resulted in a valid (acceptable) $D S_{2}$, meaning their relative error, $\Delta D S_{2} / D S_{2}$, was lower than the $1.5 \%$ threshold, for every simulation run. Further increase of the level of noise resulted in an intermediate region, in which, for the same level of noise, some of the simulations traces had valid $D S_{2}$ values whereas other traces did not. It is important to note that $\Delta D S_{2} / D S_{2}$ in these cases varied markedly between the different signals. Thus the fact that some traces where accepted while others were denied, was not a result of small fluctuations around the threshold (see Figure 1).

Further increase of the noise amplitude, resulted in unacceptable $D S_{2}$ for all the simulated signals.

Effect of $\alpha$ Sympathetic Gain, $G \alpha$ : In our previous study [3], we have found that $G_{\alpha}$ is a bifurcation parameter for the discrete model.

In the present study, we were interested in studying the effect of $G_{\alpha}$ on the $D_{2}$. More specifically we intended to investigate the region around the bifurcation. This part of the study was performed without the respiration term (equation 2), but both with and without low level noise.

As long as $G_{\alpha}$ was equal or below $20 \mathrm{msec} / \mathrm{mmHg}$, the $\mathrm{D}_{2}$ obtained were clearly lower than 2 (see Figure 2). When a small amount of noise was introduced to the model, a small but monotonic increase in $D_{2}$, as a function of $G_{\alpha}$, was observed, for $G_{\alpha}<20 \mathrm{msec} / \mathrm{mmHg}$. $G_{\alpha}$ of $21.5 \mathrm{msec} / \mathrm{mmHg}$ and above resulted in $D_{2}$ values that were above 2 .

The region of the bifurcation $\left(20<G_{\alpha}<21.5\right.$ $\mathrm{msec} / \mathrm{mmHg}$ ) was studied in more details, with

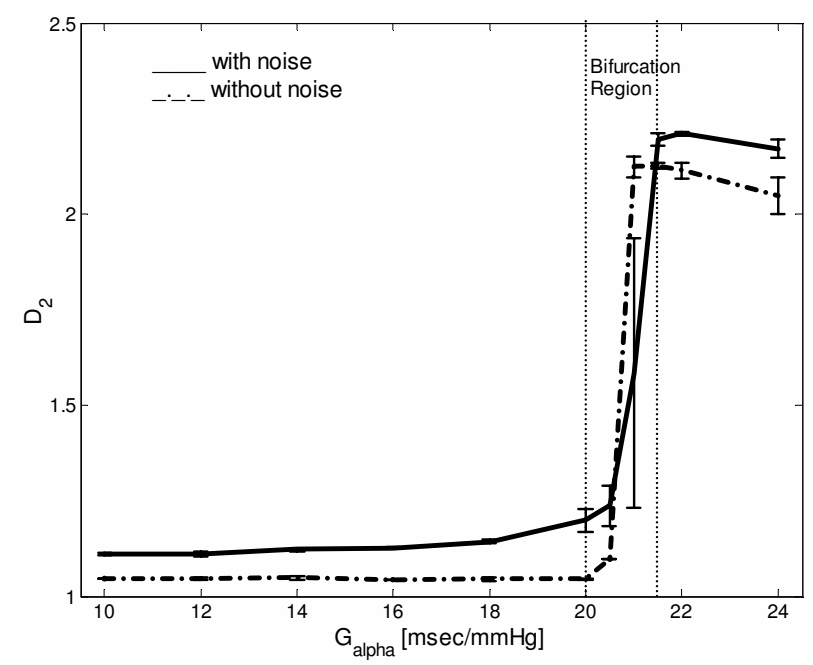

Figure 2. Correlation dimension as a function of $\alpha$ sympathetic gain.
$\Delta G_{\alpha}=0.1 \mathrm{msec} / \mathrm{mmHg}$. For the deterministic part of the simulation, the bifurcation was at $G_{\alpha}=21 \mathrm{msec} / \mathrm{mmHg}$. When a low level noise was added to the simulation, we did not obtain a clear bifurcation point for $\mathrm{G}_{\alpha}$. In the region of $21<G_{\alpha}<21.4$, we obtained that for some BP realizations, $D S_{2}$ was below 2, whereas other realizations produce values above 2 . In addition, some realizations, in this region, did not produce any valid $D S_{2}$. However when $G_{\alpha}$ reached $21.5 \mathrm{msec} / \mathrm{mmHg}$, all the realizations produced valid $D S_{2}$, all above 2 .

Effects of respiration: Changing the amplitude and/or the frequency of the respiratory term in (2), while the other parameters were set to their default values and without any noise source, also caused a bifurcation, in which $D_{2}$ jumps from below 2 to above it. Trying to obtain $D_{2}$ values larger than 3 , by combining changes that resulted in $D_{2}>2$, such as using high gain and respiratory term with post bifurcation parameters, did not succeed.

\section{Discussion}

In this work, we studied the effect of several physiological parameters on the dynamical behavior of the CVS, as reflected by $D_{2}$ calculated from computer generated BP signals.

The model that was used to produce the BP signals was a modified version of a basic beat-to-beat model of the CVS that was studied previously using linear tools $[1,2]$ and nonlinear tools [3]. The main modification in the current model with respect to [1-3] was the use of continuous BP data. The $D_{2}$ values of BP signals obtained in simulations with normal physiological parameters were in full agreement with earlier experimental studies $[4,6]$.

As expected, adding noise to the simulation increased the $D_{2}$ or destroyed the ability to calculate it. However, the addition of noise did not result in a bifurcation, indicating that the bifurcations were due to the deterministic part of the model.

In a real physiological system one should expect the presence of various non-deterministic mechanisms affecting the control of the CVS. As indicated by our results such mechanisms may affect the calculation of $D_{2}$. In this way a system with the same control parameters may show different behavior of $D_{2}(n)$ plots in two different periods of time. Evidences to such a behavior were observed in our previous experimental study [4], in which traces with high relative error were disregarded due to the suspicion that the system was non- 
stationary when these signals were recorded. According to the results in this work it can be seen that similar effect on the calculation of $D_{2}(n)$ can be observed even for traces that were generated by a model with stationary parameters.

The sensitivity of $D_{2}$ to variation in $G_{\alpha}$ was found to be very week, with the exception of the bifurcation region. Furthermore, taking into account the variation in the $D S_{2}$ values due to the noise in an experimental study indicates that it will be very hard to detect changes in $G_{\alpha}$ by calculating $D_{2}$ from experimental signals as long as it does not reach the bifurcation.

A difference of $0.5 \mathrm{msec} / \mathrm{mmHg}$ was found between the $G_{\alpha}$ values for the bifurcation, in the noise free continuous model versus the beat-to-beat model. This difference could be the result of the dominant oscillation in the HR frequency in the continuous model, which hides the small fluctuations that arise in the beat-to-beat state parameters after the bifurcation.

Studying the bifurcation as a function of $G_{\alpha}$, with the addition of a low level noise, resulted in a bifurcation region with a well-defined width as opposed to the sharp jump expected. This suggests that even low-level noise may hide the effect of the bifurcation. Once again, we see that analyzing experimental data based on calculating $D_{2}$ from BP signal, may be very complicated due to the noise. Nonetheless, the noise does not eliminate completely the effect of the bifurcation. Furthermore, since the bifurcation region of the noisy signal does not start before the actual noise-free bifurcation occurs, it might be possible, even in a noisy experiment, to obtain an upper bound for the onset of the noise free bifurcation.

Another result seen in the study of the effect of $G_{\alpha}$ on the dynamics was that the simulated BP signal that was produced with the post bifurcation $G_{\alpha}$, showed enhanced oscillation at $0.1 \mathrm{~Hz}$, known as Mayer waves. These findings are in agreement with [7], which also suggested that the Mayer waves could be explained as gain induced oscillations.

In conclusion, we have shown that even a simple model of the CVS exhibits dynamical behavior, which is characteristic to nonlinear systems. Many parameters such as: level of noises, gains, and respiration parameters, were shown to have an effect on $D_{2}$. However the sensitivity of $D_{2}$ to variations in these parameters was found to be low, in exclusion of the range of bifurcation transition. This low sensitivity results in a poor signal to noise ratio in real life studies, which leads us to the conclusion that the ability to gain physiological insight about the CVS by calculating $\mathrm{D}_{2}$ for the BP signal, is very limited. Nonetheless since the system has displayed obvious signs of nonlinearity, it is important to develop a good understanding of its nonlinear features.

\section{Acknowledgements}

This study was supported by a grant from the Israeli Science Foundation and by the Abramson Foundation.

\section{Reference}

[1] DeBoer RW, Karemaker JM, Strackee J. Hemodynamic fluctuations and baroreflex sensitivity in humans: a beatto-beat model. American Journal of Physiology-Heart and Circulatory Physiology 1987; 253:680-689.

[2] Whittam AM, Clayton RH, Lord SW, McComb JM, Murray A. Heart rate and blood pressure variability in normal subjects compared with data from beat-to-beat models developed from de Boer's model of the cardiovascular system. Physiological Measurement 2000; 21(2):305-318.

[3] Eyal S, Akselrod S. Bifurcation in a simple model of the cardiovascular system. Methods of Information in Medicine 2000; 39(2):118-121.

[4] Eyal S, Almog $\mathrm{Y}, \mathrm{Oz} \mathrm{O}$, Eliash S, Akselrod S. Nonlinear dynamics applied to blood pressure control. Autonomic Neuroscience-Basic \& Clinical 2001; 89(12):24-30.

[5] Grassberger P, Procaccia I. Measuring the strangeness of strange attractors. Physica D 1983; 9:189-208.

[6] Mrowka R, Stauss HM, Wagner CD, Nafz B, Patzak A, Persson PB. Non Linear analysis of the cardiovascular control system in rat strains with differing hemodynamic characteristics. In: Murry A, Arzbaecher R, editors. Comupers in Cardiology 1995. Piscataway, NJ USA: IEEE, 1995:313-5.

[7] Abbiw-Jackson RM, Langford WF. Gain-induced oscillations in blood pressure. Journal of Mathematical Biology 1998; 37(3):203-234.

Address for correspondence.

Solange Akselrod.

School of Physics and Astronomy

Tel-Aviv University, Tel-Aviv, 69978 Israel.

E-mail: solange@post.tau.ac.il 